\section{Plasma cell proliferative index predicts outcome in immunoglobulin light chain amyloidosis treated with stem cell transplantation}

\author{
M. Hasib Sidiqi, ${ }^{1}$ Mohammed A. Aljama, ${ }^{1}$ Dragan Jevremovic, ${ }^{2}$ \\ William G. Morice, ${ }^{2}$ Michael Timm, ${ }^{2}$ Francis K. Buadi, ${ }^{1}$ Rahma Warsame, ${ }^{1}$ \\ Martha Q. Lacy, ${ }^{1}$ Angela Dispenzieri, ${ }^{1}$ David Dingli, ${ }^{1}$ Wilson I. Gonsalves, ${ }^{1}$ \\ Shaji Kumar, ${ }^{1}$ Prashant Kapoor, ${ }^{1}$ Taxiarchis Kourelis, ${ }^{1}$ Nelson Leung, ${ }^{3}$ \\ William J. Hogan ${ }^{1}$ and Morie Gertz ${ }^{1}$
}

${ }^{1}$ Division of Hematology, Department of Internal Medicine; ${ }^{2}$ Department of Laboratory Medicine and Pathology and ${ }^{3}$ Division of Nephrology and Hypertension, Mayo Clinic, Rochester, MN, USA
Haematologica 2018

Volume 103(7):1229-1234

\title{
ABSTRACT
}

T he plasma cell proliferative index provides an insight into plasma cell biology in plasma cell disorders and is an important prognostic marker in myeloma and smoldering myeloma. We analyzed the prognostic impact of the plasma cell proliferative index in 513 patients with systemic immunoglobulin light chain (AL) amyloidosis undergoing stem cell transplantation at the Mayo Clinic between $1^{\text {st }}$ January 2003 and $31^{\text {st }}$ August 2016. Two cohorts were identified according to Low or Elevated plasma cell proliferative index. Patients with an Elevated plasma cell proliferative index had more cardiac involvement $(56 \% \mathrm{vs} .44 \%$; $P=0.01)$, less renal involvement (55\% vs. $70 \% ; P=0.001)$, and were more likely to have $10 \%$ or over bone marrow plasma cells (58\% vs. $32 \%$; $P<0.0001)$ compared to those with a Low plasma cell proliferative index. Both progression-free survival and overall survival were lower in patients with an Elevated compared to Low plasma cell proliferative index: median progression-free survival 44 vs. 95 months $(P<0.0001)$ and median overall survival 102 vs. 143 months $(P=0.0003)$. All-cause mortality at 100 days was higher in patients with an Elevated plasma cell proliferative index (elevated $10.3 \%$ vs. low $4.3 \% ; P=0.008$ ). On multivariate analysis Elevated plasma cell proliferative index was an independent prognostic factor for overall survival (Hazard Ratio 1.5, 95\% CI: 1.1-2.1; $P=0.021$ ). The plasma cell proliferative index is an important prognostic tool in patients with AL amyloidosis undergoing stem cell transplant.

\section{Introduction}

Immunoglobulin light chain (AL) amyloidosis is a multi-system disorder characterized by a plasma cell or B-cell clone producing misfolded light chain proteins that deposit in tissues causing tissue damage and organ dysfunction. ${ }^{1}$ Patients with AL amyloidosis typically have a low tumor burden with the majority of patients having bone marrow plasma cells (BMPC) of less than $10 \%$ at diagnosis. ${ }^{2}$ The plasma cell proliferative index (PCPI) provides an insight into plasma cell biology in plasma cell disorders. It recognizes cells that are actively synthesizing DNA and gives an indication of the proliferative rate of the malignant plasma cells. The bone marrow PCPI has been identified as a strong prognostic marker in both active multiple myeloma and smoldering myeloma. ${ }^{3.5}$ Data regarding its utility in AL amyloidosis are scant, with an early report suggesting patients with an elevated PCPI had a worse overall survival. ${ }^{6}$ However, this pre-dated the use of autologous stem cell transplant (ASCT) therapy for AL amyloidosis and the advent of novel therapies as therapeutic options for patients with AL amyloidosis.

\section{Correspondence:}

gertz.morie@mayo.edu

Received: January 30, 2018.

Accepted: April 16, 2018.

Pre-published: April 19, 2018.

doi:10.3324/haematol.2018.189985

Check the online version for the most updated information on this article, online supplements, and information on authorship \& disclosures: www.haematologica.org/content/103/71229

\section{(C)2018 Ferrata Storti Foundation}

Material published in Haematologica is covered by copyright. All rights are reserved to the Ferrata Storti Foundation. Use of published material is allowed under the following terms and conditions:

https://creativecommons.org/licenses/by-nc/4.0/legalcode. Copies of published material are allowed for personal or internal use. Sharing published material for non-commercial purposes is subject to the following conditions:

https://creativecommons.org/licenses/by-nc/4.0/legalcode, sect. 3. Reproducing and sharing published material for commercial purposes is not allowed without permission in writing from the publisher. 
We review the prognostic impact of the plasma cell proliferative index in a large cohort of patients with AL amyloidosis undergoing stem cell transplant at the Mayo Clinic, and assess patients' and disease characteristics as well as the prognostic impact of PCPI on outcome and survival.

\section{Methods}

After approval by the Mayo Clinic Institutional Review Board, data were reviewed on all patients with biopsy proven systemic AL amyloidosis who underwent autologous stem cell transplant between $1^{\text {st January }} 2003$ and $31^{\text {st }}$ August 2016 and had a plasma

Table 1. Patients' baseline characteristics.

\begin{tabular}{|c|c|c|c|}
\hline Characteristic & Low PCPI (n=348) & Elevated PCPI (n=165) & $P$ \\
\hline Age, years, median (IQR) & $59(53-65)$ & $58(52-63)$ & 0.26 \\
\hline Male $\%$ & 64 & 63 & 0.77 \\
\hline \multicolumn{4}{|l|}{ Organs involved, $\mathrm{n}(\%)$} \\
\hline Cardiac & $153(44)$ & $93(56)$ & 0.01 \\
\hline Renal & $243(70)$ & $90(55)$ & 0.001 \\
\hline Hepatic & $35(10)$ & $19(11)$ & 0.65 \\
\hline Neurological & $49(14)$ & $25(15)$ & 0.79 \\
\hline Other & $76(22)$ & $52(31)$ & 0.02 \\
\hline Organs involved, $\mathrm{n}(\%)$ & & & 0.12 \\
\hline 1 & $164(47)$ & $62(37)$ & \\
\hline 2 & $124(36)$ & $69(42)$ & \\
\hline$\geq 3$ & $60(17)$ & $34(21)$ & \\
\hline Light chain, $\mathrm{n}(\%)$ & & & 0.52 \\
\hline Lambda & $258(74)$ & $118(72)$ & \\
\hline Карра & $90(26)$ & $47(28)$ & \\
\hline Pre-ASCT light chain mg/dL, median (IQR) & $12.4(5.8-34.4)$ & $20.8(8.7-56.3)$ & 0.0004 \\
\hline Bone marrow plasma cells, n (\%) & & & $<0.0001$ \\
\hline$<10 \%$ & $236(68)$ & $70(42)$ & \\
\hline$\geq 10 \%$ & $112(32)$ & $95(58)$ & \\
\hline Creatinine, median, mg/dL & $1(0.9-1.3)$ & $1(0.9-1.2)$ & 0.88 \\
\hline Urine 24-hour protein, median, $g$ & $3.3(0.2-7.0)$ & $0.98(0.2-6.1)$ & 0.049 \\
\hline NT-Pro BNP, median, pg/mL (IQR) & $417(150-1757)$ & $901(197-2499)$ & 0.02 \\
\hline NT-Pro BNP > 8500, n (\%) & $13(4 \%)$ & $3(2 \%)$ & 0.28 \\
\hline Troponin T, median, ng/mL (IQR) & $0.01(0.01-0.02)$ & $0.01(0.01-0.03)$ & 0.13 \\
\hline Mayo Stage 2004, n (\%) & & & 0.38 \\
\hline I & $200(68)$ & $91(61)$ & \\
\hline II & $46(16)$ & $29(20)$ & \\
\hline III & $48(16)$ & $28(19)$ & \\
\hline Missing & 54 & 17 & \\
\hline Mayo Stage 2012, n (\%) & & & 0.03 \\
\hline I & $139(48)$ & $51(35)$ & \\
\hline II & 79 (27) & $54(37)$ & \\
\hline III & $45(16)$ & $22(15)$ & \\
\hline IV & $26(9)$ & $20(13)$ & \\
\hline Missing & 59 & 18 & \\
\hline Conditioning, n (\%) & & & 0.02 \\
\hline Melphalan 200 mg/m² & $256(74)$ & $104(63)$ & \\
\hline Melphalan $<200 \mathrm{mg} / \mathrm{m}^{2}$ & $89(26)$ & $60(37)$ & \\
\hline Pre-ASCT chemotherapy n (\%) & & & 0.0023 \\
\hline Untreated & $221(64)$ & $78(47)$ & 0.0006 \\
\hline Corticosteroid only & $35(10)$ & $19(12)$ & \\
\hline Melphalan based & $14(4)$ & $15(9)$ & \\
\hline IMID based & $19(5)$ & $16(10)$ & \\
\hline Bortezomib based & $48(14)$ & $35(21)$ & \\
\hline Other & $11(3)$ & $2(1)$ & \\
\hline Time Period of Transplant, $\mathrm{n}(\%)$ & & & 0.5 \\
\hline$<2010$ & $207(59)$ & $93(56)$ & \\
\hline$\geq 2010$ & $141(41)$ & $72(44)$ & \\
\hline
\end{tabular}

ASCT: autologous stem cell transplant; PCPI: plasma cell proliferation index; IMiD: immunomodulatory drug: IQR: interquartile range; n: number. 

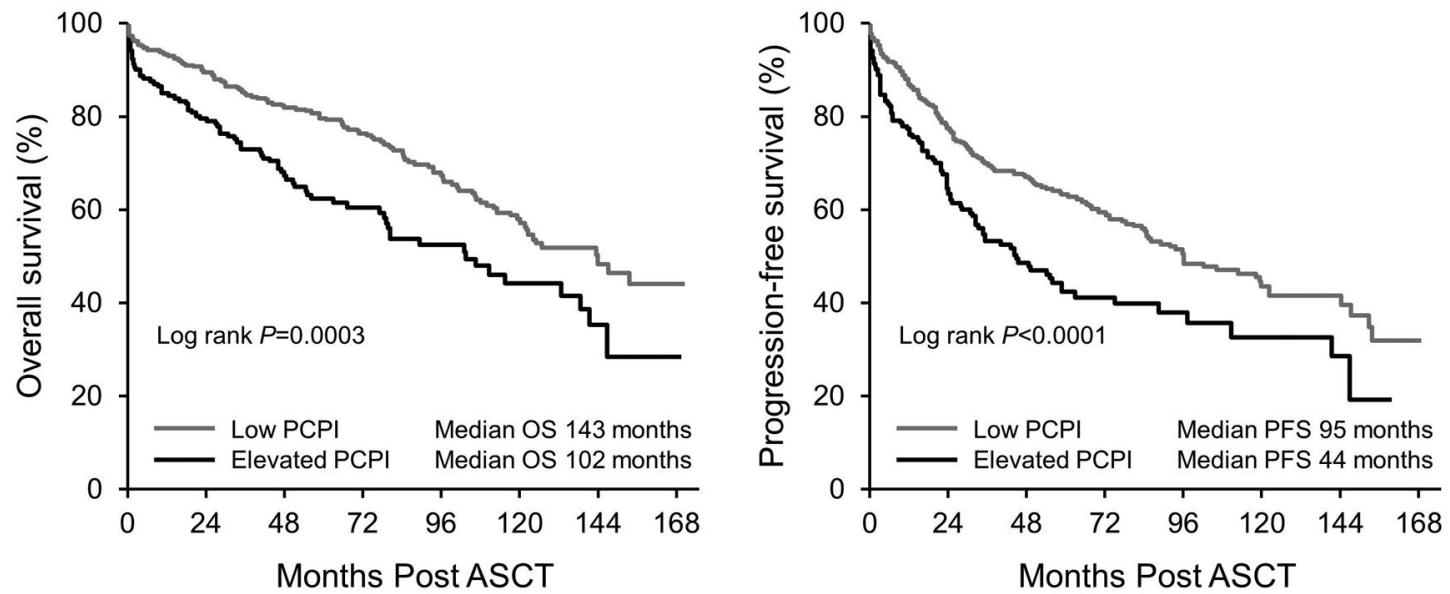

N. at risk

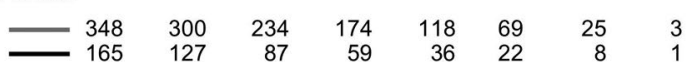

N. at risk

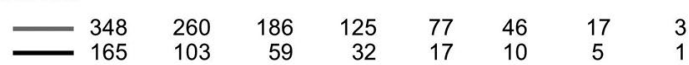

Figure 1. Overall survival and progression-free survival. PCPI: plasma cell proliferation index; ASCT: autologous stem cell transplant; PFS: progression-free survival; OS: overall survival.

Table 2. Hematologic response.

\begin{tabular}{lcc} 
& Low PCPI n (\%) & Elevated PCPI n (\%) \\
CR & & \\
VGPR & $150(44)$ & $63(40)$ \\
PR & $101(29)$ & $49(31)$ \\
NR & $48(14)$ & $25(16)$ \\
NA & $44(13)$ & $21(13)$ \\
\hline
\end{tabular}

CR: complete response;VGPR: very good partial response; PR: partial response; NR: no response; NA: not available; PCPI: plasma cell proliferation index.

cell proliferative index (PCPI) performed on bone marrow samples at diagnosis. The PCPI is expressed as a percentage of plasma cells in $S$ phase. Patients with insufficient plasma cells identified for an accurate assessment of the PCPI were excluded.

Prior to May of 2012, the plasma cell proliferative index was measured using the bromodeoxyuridine (BrdU) method described previously. ${ }^{4}$ From May 2012 onwards, the BrdU method was replaced by the DNA content measurement using a flow cytometry technique and a summary of this method is available in the Online Supplementary Appendix.

We initially analyzed all patients with a PCPI performed using the BrdU method ( $\mathrm{n}=390)$. The median PCPI was $0 \%$ with at least two-thirds (69\%) of patients having a PCPI of $0 \%$. Amongst the patients with a PCPI performed using the flow cytometric method $(n=123)$, the median PCPI was $0.3 \%$ with approximately two-thirds $(65 \%)$ of patients having a PCPI of $0.5 \%$ or under. To account for differences in the sensitivity of the methods, we established "Low" PCPI as $0 \%$ by the BrdU method and $0.5 \%$ or under by the flow cytometry method. "Elevated" PCPI was defined as over $0 \%$ by the BrdU method and over $0.5 \%$ by the flow cytometric method. This established two cohorts of patients: those with a Low PCPI $(n=348)$ and those with an Elevated PCPI $(n=165)$. Patients', disease and treatment characteristics as well as outcomes were assessed between the two groups.
Organ involvement and hematologic response were assessed according to consensus criteria., ${ }^{7,8}$ Risk stratification was according to the Mayo 2012 and 2004 staging systems., ${ }^{9,10}$ Patients were selected for ASCT using available criteria at the time of transplant. Patients were mobilized, conditioned and transplanted according to previously published institutional protocols. Response was measured at approximately 100 days post ASCT according to up-dated consensus criteria. Overall response rate (ORR) was defined as a partial response (PR) or greater.

Statistical analysis was performed on JMP software (SAS, Cary, NC, USA). Patients' and disease related factors were compared using the $\chi^{2}$ test for categorical variables and the Wilcoxon signed rank test for continuous variables. Survival analysis was performed using the Kaplan-Meier method. Overall survival (OS) was calculated from day 0 of bone marrow transplant to death from any cause. All-cause mortality at 100 days was defined as death from any cause within 100 days post ASCT. The Cox proportional hazards model was used to assess for predictors of OS. The variables included in the univariate analysis were age, sex, number of organs involved, bone marrow plasma cells, Mayo Stage, conditioning dose, pre-transplantation chemotherapy, and PCPI. Variables reaching a $P<0.1$ were included in the multivariate analysis. 


\section{Hematologic Response}

\section{Low PCPI}

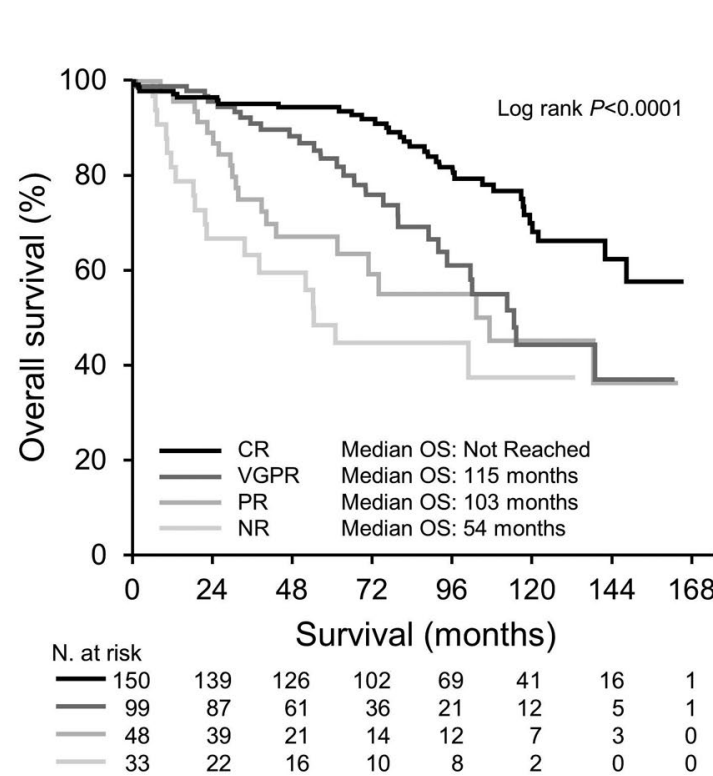

\section{Elevated PCPI}

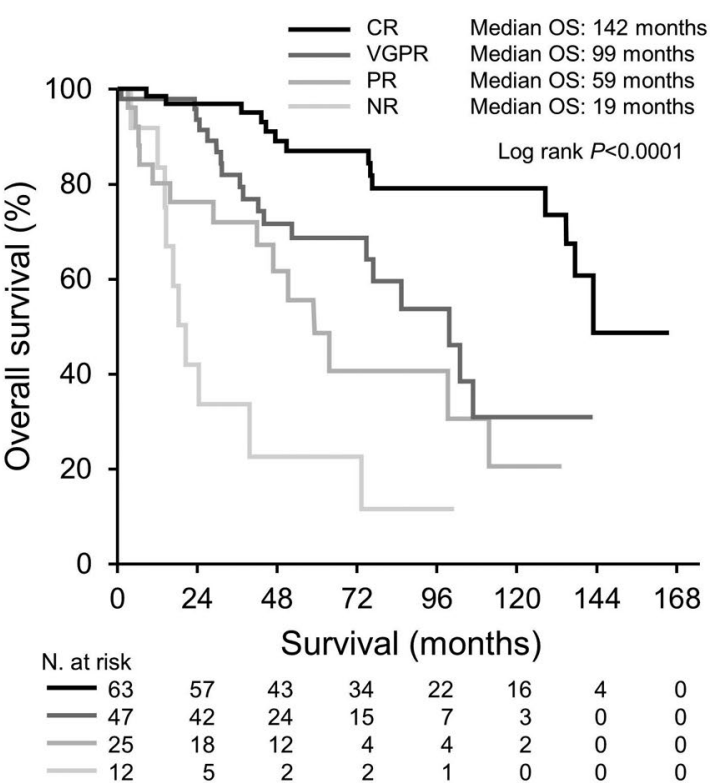

Figure 2. Landmark survival analysis from 100 days post stem cell transplant by hematologic response. CR: complete response; VGPR: very good partial response; PR: partial response; NR: no response; PCPI: plasma cell proliferation index; OS: overall survival; N: number.

Table 3. Prognostic factors for survival.

\begin{tabular}{|c|c|c|c|c|c|c|}
\hline \multirow[t]{2}{*}{ Variable } & \multicolumn{2}{|c|}{ Univariate } & \multicolumn{2}{|c|}{ Multivariate Model A } & \multicolumn{2}{|c|}{ Multivariate Model B } \\
\hline & $P$ & HR & $P$ & HR & $P$ & HR \\
\hline Age $\geq 65$ & 0.0074 & $1.6(1.1-2.1)$ & NS & - & NS & - \\
\hline Male & 0.014 & $1.4(1.1-2.0)$ & NS & - & NS & - \\
\hline BMPCs $\geq 10$ & 0.0033 & $1.5(1.2-2.1)$ & NS & - & NS & - \\
\hline PCPI elevated & 0.0005 & $1.7(1.3-2.3)$ & 0.021 & $1.5(1.1-2.1)$ & 0.019 & $1.5(1.1-2.1)$ \\
\hline Mayo stage 2012 III/IV vs. I/II & $<0.0001$ & $3.5(2.5-4.8)$ & $<0.0001$ & $2.3(1.6-3.3)$ & Not included & - \\
\hline Mayo Stage 2004 III vs. I/II & $<0.0001$ & $0.3(0.2-0.4)$ & Not included & - & 0.0006 & $2.0(1.3-2.8)$ \\
\hline Conditioning dose 200 vs. $<200$ & $<0.0001$ & $0.3(0.3-0.5)$ & $<0.0001$ & $0.4(0.3-0.6)$ & $<0.0001$ & $0.4(0.3-0.6)$ \\
\hline
\end{tabular}

BMPCs: bone marrow plasma cells; PCPI: plasma cell proliferation index; NS: not significant; HR: Hazard Ratio.

\section{Results}

Between $1^{\text {st January }} 2003$ and $31^{\text {st }}$ of August 2016548 patients with systemic AL amyloidosis underwent ASCT at the Mayo Clinic in Rochester, for $94 \%$ of whom $(n=513)$ data were available on bone marrow PCPI performed at diagnosis and $6 \%(n=35)$ had insufficient plasma cells on bone marrow biopsy for accurate assessment of PCPI. Of those with a reported PCPI, $68 \%(n=348)$ had a Low PCPI and 32\% ( $n=165)$ had an Elevated PCPI. Table 1 summarizes the baseline characteristics for each group of patients. Median age and proportion of males in the Low PCPI group was similar to those with an Elevated PCPI: 59 vs. 58 years $(P=0.26)$ and $64 \%$ vs. $63 \%$ $(P=0.77)$, respectively.

Patients with an Elevated PCPI had more cardiac involvement ( $56 \%$ vs. $44 \% ; P=0.01)$ and less renal involvement $(55 \%$ vs. $70 \% ; P=0.001)$. The number of organs involved was similar between the two groups. Bone marrow plasma cell burden was higher in the Elevated PCPI group with $58 \%$ of patients having BMPCs of $10 \%$ or over compared to $32 \%$ in the Low PCPI group $(P<0.0001)$. A higher plasma cell percentage would be expected in the former group given the higher proliferative capacity of the plasma cell clone. More patients had a Mayo Stage of II or more in the Elevated PCPI group (65\% elevated PCPI vs. 52\% low PCPI; $P=0.008$ ). Fewer patients in the Elevated PCPI group received full intensity (melphalan $200 \mathrm{mg} / \mathrm{m}^{2}$ ) conditioning (63\% Elevated PCPI vs. 74\% Low PCPI; $P=0.02)$. Reduced intensity conditioning (melphalan $<200 \mathrm{mg} / \mathrm{m}^{2}$ ) was given to patients aged 70 years or older and those with creatinine over $1.8 \mathrm{mg} / \mathrm{dL}$. More patients received pre-transplant chemotherapy in the Elevated PCPI group 


\section{Mayo Stage 2012}

Low PCPI

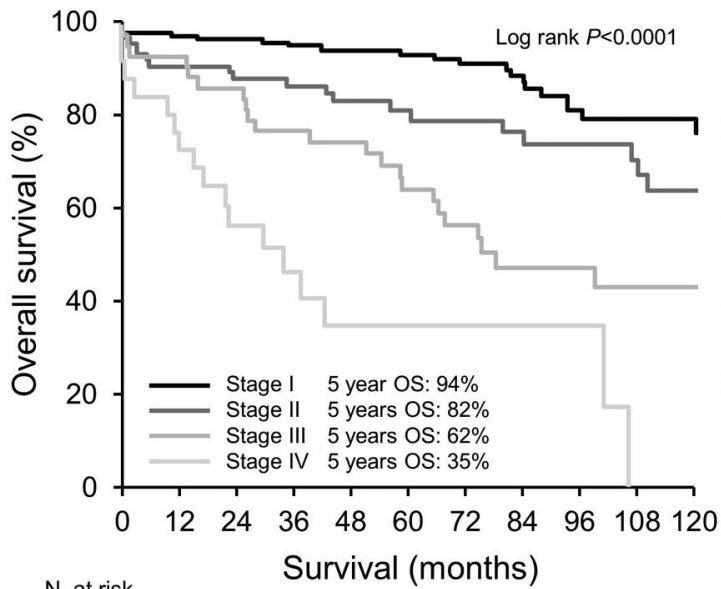

Elevated PCPI

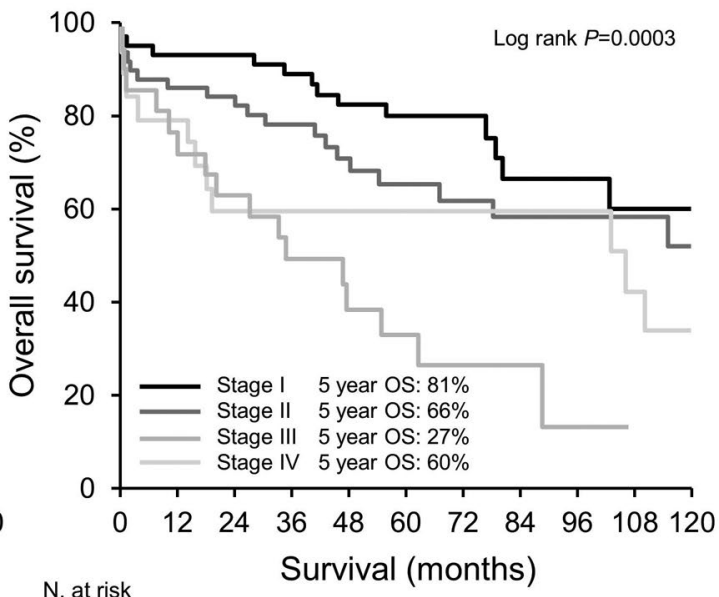

\begin{tabular}{rrrrrr} 
N. at risk & \multicolumn{5}{c}{} \\
\hline 139 & 133 & 108 & 77 & 46 & 25 \\
-79 & 63 & 48 & 32 & 25 & 13 \\
-45 & 37 & 30 & 21 & 13 & 7 \\
26 & 13 & 6 & 4 & 2 & 0
\end{tabular}

$36 \quad 21$

$\begin{array}{rl}10 & 6 \\ 13 & 7 \\ 1 & 0 \\ 7 & 3\end{array}$

Figure 3. Mayo Stage 2012. Survival by Mayo Stage 2012. PCPI: plasma cell proliferation index; OS: overall survival; N: number.

compared to the Low PCPI cohort (53\% vs. 36\%; $P=0.0006)$. This may reflect our policy of giving pretransplantation chemotherapy induction in patients with more than $10 \%$ plasma cells in their bone marrow at diagnosis.

Data regarding response were available in 501 of 513 $(98 \%)$ of patients and are summarized in Table 2. Overall response rate was similar in both groups $(87 \%$ in Elevated PCPI and $87 \%$ in Low PCPI; $P=0.86)$ as were rates of complete response (40\% in Elevated PCPI and $44 \%$ in Low PCPI; $P=0.44)$. In the Low PCPI group, the ORR was not affected by pre-transplantation chemotherapy: $86 \%$ in untreated patients and $90 \%$ in treated patients $(P=0.4)$. For patients with an Elevated PCPI, the ORR was significantly higher in patients receiving pretransplantation chemotherapy than those who did not ( $92 \%$ vs. $74 \%$, respectively; $P=0.003$ ).

Median PFS and OS for the entire cohort were 84 and 126 months, respectively. Both PFS and OS were lower in patients with an Elevated PCPI compared to Low PCPI (median PFS 44 vs. 95 months, $P<0.0001$, and median $O S$ 102 vs. 143 months, $P=0.0003$, respectively) (Figure 1). Hematologic response (Figure 2) and Mayo Stage (Figure 3) predicted survival. In the Elevated PCPI cohort, those with Mayo Stage IV appeared to do better than those with Mayo Stage III; however, we believe this is likely to be due to the small numbers in the two groups ( $n=20$ and 22 , respectively). Chemotherapy prior to transplantation did not impact OS in either cohort (Low PCPI Pre-ASCT chemotherapy median OS 143 months vs. Untreated median OS 142 months, $P=0.65$, and PCL over 0 PreASCT chemotherapy median OS 102 months vs. Untreated median OS 114 months, $P=0.86$ ). All-cause mortality at 100 days was significantly higher in patients
100 Day Mortality

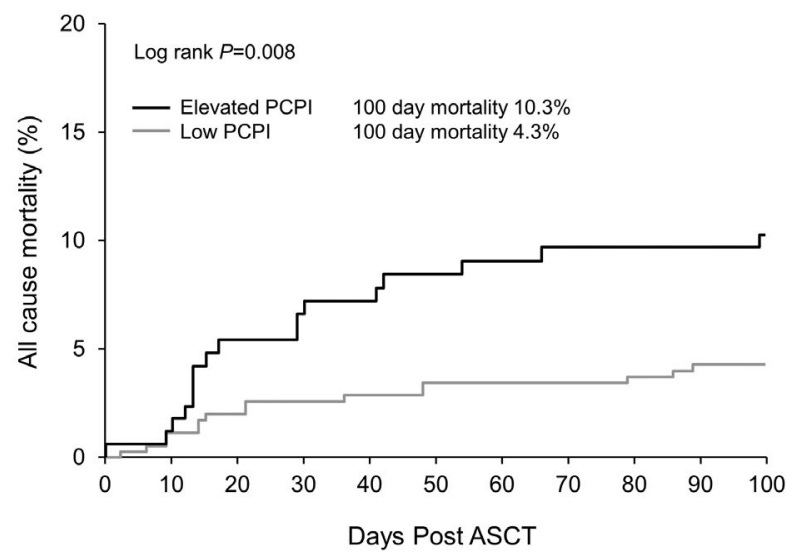

Figure 4. Day 100 all-cause mortality. PCPI: plasma cell proliferation index; ASCT: autologous stem cell transplant.

with an Elevated PCPI compared to those with a Low PCPI (10.3\% vs. $4.3 \%$; $P=0.008$ ) (Figure 4$)$.

Table 3 shows the results for predictors of OS by univariate and multivariate analysis. On univariate analysis age, male sex, BMPCs $10 \%$ or over, Elevated PCPI, Mayo Stage, and conditioning dose were all prognostic factors for OS. On multivariate analysis the only independent prognostic factors for OS were Mayo Stage, conditioning dose (melphalan 200 vs. melphalan less than 200) and an Elevated PCPI (Hazard Ratio 1.5, 95\% CI: 1.1-2.1; $P=0.02$ ). 


\section{Discussion}

Increased plasma cell proliferation rate has been recognized as a predictor of a more aggressive clinical course in patients with myeloma and amyloidosis. ${ }^{3-6,11}$ The early studies of plasma cell proliferation rate were carried out with $\mathrm{BrDU}$ incorporation and microscopic review of plasma cells on the slide. However, flow cytometric evaluation of proliferation index is much more robust, precise and reproducible. ${ }^{11}$ In addition, since flow cytometry immunophenotying is already the standard of care for the diagnosis of plasma cell neoplasms, incorporation of proliferation measurement by DAPI staining is an easy addition to currently run assays, and therefore readily available for any clinical laboratory to perform. Since all patients with light chain amyloidosis require a bone marrow for assessment, ample material should be available for direct measurement of the proliferative index at time of diagnosis.

The proliferative index is an independent predictor of survival and adds importantly to what is currently available based on the pre-transplantation variable of Mayo Stage as well as the post hoc variables of depth of response and conditioning dose of chemotherapy. We note that patients with an elevated PCPI had more cardiac involvement and a higher pre-transplant light chain level. Despite this, elevated PCPI remained an independent predictor of survival in a multivariate model that included the powerful prognostic factor of Mayo Stage, a variable that incorporates both cardiac biomarkers and light chain level in staging patients with AL amyloidosis. Pre-transplantation chemotherapy was associated with an improved overall response rate in patients with an elevated PCPI but not those with a low PCPI. Therefore the plasma cell proliferative index may be used as a potential guide to identify patients that may benefit most from pre-transplantation chemotherapy, suggesting that patients with an Elevated labeling index should be strongly considered for induction chemotherapy prior to transplantation. In addition, an Elevated PCPI predicts earlier progression, suggesting that consolidation chemotherapy or maintenance post transplant should be considered for this patient population. Given that hematologic response is a strong predictor of survival, achieving deeper responses in this cohort may help improve outcomes.

Our data also reveal higher 100-day mortality in patients with an Elevated PCPI. Patients with an Elevated PCPI were more likely to have cardiac involvement and a higher Mayo Stage; both have been associated with a worse prognosis. ${ }^{10,12}$ In addition, patients with an Elevated PCPI were more likely to receive chemotherapy, with potential toxicity, prior to transplantation.

Our study is limited by its retrospective nature and potentially by a change in the method of testing for the plasma cell proliferation index during the study time period. We have tried to account for the difference in assay sensitivity by establishing unique cut offs for elevated PCPI for the two methods. In addition, our study includes patients over a time period of almost 14 years and selection criteria for stem cell transplant eligibility in AL amyloidosis have significantly changed over this time. Despite these limitations, the significance of the labeling index on survival independent of previously known factors is an important addition to the prognosis of patients with this serious disorder.

\section{References}

1. Merlini G, Seldin DC, Gertz MA. Amyloidosis: pathogenesis and new therapeutic options. J Clin Oncol. 2011;29(14):1924-1933.

2. Kourelis TV, Kumar SK, Gertz MA, et al. Coexistent multiple myeloma or increased bone marrow plasma cells define equally high-risk populations in patients with immunoglobulin light chain amyloidosis. J Clin Oncol. 2013;31(34):4319-4324.

3. Madan S, Kyle RA, Greipp PR. Plasma cell labeling index in the evaluation of smoldering (asymptomatic) multiple myeloma. Mayo Clin Proc. 2010;85(3):300.

4. Kumar S, Rajkumar SV, Greipp PR, Witzig TE. Cell proliferation of myeloma plasma cells: comparison of the blood and marrow compartments. Am J Hematol. 2004; 77(1):7-11.

5. Greipp PR, Lust JA, O'Fallon WM, Katzmann JA, Witzig TE, Kyle RA. Plasma cell labeling index and beta 2-microglobu- lin predict survival independent of thymidine kinase and C-reactive protein in multiple myeloma. Blood. 1993;81(12):33823387.

6. Gertz MA, Kyle RA, Greipp PR. The plasma cell labeling index: a valuable tool in primary systemic amyloidosis. Blood. 1989;74(3):1108-1111.

7. Gertz MA, Comenzo R, Falk RH, et al. Definition of organ involvement and treatment response in immunoglobulin light chain amyloidosis (AL): a consensus opinion from the 10th International Symposium on Amyloid and Amyloidosis, Tours, France, 18-22 April 2004. Am J Hematol. 2005;79(4):319-328.

8. Palladini G, Dispenzieri A, Gertz MA, et al. New criteria for response to treatment in immunoglobulin light chain amyloidosis based on free light chain measurement and cardiac biomarkers: impact on survival outcomes. J Clin Oncol. 2012;30(36):45414549.

9. Dispenzieri A, Gertz MA, Kyle RA, et al.
Serum cardiac troponins and N-terminal pro-brain natriuretic peptide: a staging system for primary systemic amyloidosis. J Clin Oncol. 2004;22(18):3751-3757.

10. Kumar S, Dispenzieri A, Lacy MQ, et al. Revised prognostic staging system for light chain amyloidosis incorporating cardiac biomarkers and serum free light chain measurements. J Clin Oncol. 2012; 30(9):989-995.

11. Paiva B, Vidriales MB, Montalban MA, et al. Multiparameter flow cytometry evaluation of plasma cell DNA content and proliferation in 595 transplant-eligible patients with myeloma included in the Spanish GEM2000 and GEM2005<65y trials. Am J Pathol. 2012;181(5):1870-1878.

12. Dispenzieri A, Gertz MA, Kyle RA, et al. Prognostication of survival using cardiac troponins and $\mathrm{N}$-terminal pro-brain natriuretic peptide in patients with primary systemic amyloidosis undergoing peripheral blood stem cell transplantation. Blood. 2004;104(6):1881-1887. 